in women, suggesting an oestrogen withdrawal hypothesis in these disorders (Deuchar \& Brockington, 1998). Oestrogen release into the peripheral blood is influenced by secretion of the pituitary hormone gonadorelin. We describe a psychotic disorder associated with administration of a long-acting luteinising hormone-releasing hormone agonist (LALHRHA) triptorelin.

Mrs A., 32 years old with two children, had been receiving monthly treatment for 2 months with triptorelin (intramuscular depot formulation: $3 \mathrm{mg} / 4$ weeks) for suspected endometriosis. A few days after the second injection, she experienced persecutory delusions, agitation, and auditory hallucinations, which resolved within two months under neuroleptic medication and were immediately followed by a major depressive episode. This episode fulfilled DSM-IV (American Psychiatric Association, 1994) criteria for schizoaffective disorder. There was no personal history of psychotic disorder (mild baby blues followed the birth of the first child) and no familial history of psychiatric disorder. One year after this episode, she became pregnant. There were no psychiatric symptoms during the pregnancy. Five days after the delivery she presented in a psychotic state clinically similar to the previous one.

Peripheral oestrogen modulates dopaminergic transmission (Fink et al, 1996). The oestrogen decrease has been hypothesised as being a causative factor in puerperal and premenstrual psychoses in predisposed individuals (Deuchar \& Brockington, 1988). LALHRHAs administered in a depot formulation induce a paradoxical desensitisation of pituitary gonadotrophin secretion, resulting in a reduction in secretion of luteinising hormone and follicle stimulating hormone, and consequently a reduction in oestrogen secretion within one week (Broekmans et al, 1992).

As observed in puerperal and premenstrual psychoses, the decrease in oestrogen levels induced by the administration of LALHRHAs is consistent with a modification in the brain oestrogen environment, and is simultaneously associated with the occurrence of acute psychosis. The occurrence of both puerperal psychosis (suggesting an individual predisposition) and LALHRHAassociated psychosis in the same individual suggests a common aetiology. The puerperal and the LALHRHA-associated psychosis showed clinical and hormonal similarities, suggesting that a hormonal mechanism (the delivery-induced and the LALHRHA- induced oestrogen decrease) might be aetiologically relevant in this patient.

American Pyychiatric Association (1994) Diognostic and Statistical Manual of Mentol Disorders (4th edn) (DSM-I). Washington, DC: APA.

Broekcuans, E. J., Bernardus, R. E., Berkhout, G., ct al (1992) Pituitary and ovarian suppression after early follicular and mid-luteal administration of a LHRH agonist in depot formulation: decapeptyl CR. Gynecological Endocrinology، 6, 153-161.

Deuchar, N. \& Brockington, I. (1998) Puerperal and menstrual psychoses: the proposal of a unitary etiological hypothesis. Journal of Psychosomatic Obstetrics and Gynoecology, 19, 104-110.

Fink, G., Sumner, B. E., Rosie, R., et of (1996) Estrogen control of central neurotransmission: effect on mood, mental state, and memory. Cellular and Molecular Neurobiology, 16, 325-344.

V. Mahe, J. Nartowski, F. Montagnon,

A. Dumaine, N. Glück Service de Psychiatrie, Hôpital Général de Meaux, 6-8 rue Saint-Fiacre. BP218, 77104 Meaux cedex, France

\section{Manic episode due to gabapentin treatment}

Sir: Short \& Cooke (1995) reported on a case of hypomania associated with gabapentin treatment. In a comment on this report Hauck \& Bhaumik (1995) felt that alternating psychosis might be the cause (though electroencephalogram (EEG) was lacking). We report on a 35 -year-old woman with a manic episode under gabapentin treatment in whom alternating psychosis as a possible causal factor could be excluded. She suffered from complex partial and tonicclonic seizures for at least three years. The magnetic resonance imaging scan revealed glio-neuronal hamartia both in the left hippocampus and the right amygdala. A gabapentin monotherapy (3200 mg/day) was achieved five months before admission. Seizure frequency and EEG (within normal range) remained unchanged, however, the patient suffered from labile mood around menstruation and reported a slightly elevated mood in general.

Elevated mood and increased activity with brief episodes of depression over two days led to the patient's admission to hospital. Speech was slightly accelerated. The initial score on the Bech-Rafaelsen Mania scale (BRMAS; Bech et al, 1978) was 23. Neurological examination and EEG remained normal. Epileptic seizures had not occurred before admission. She did not have a personal or a family history of psychiatric disorders. Psychiatric symptoms disappeared within five days after discontinuation of gabapentin (BRMAS=5). At that time an anticonvulsant and antimanic treatment with lorazepam (2 mg/ day) and oxcarbazepine (1200 mg/day) was established.

Psychic reactions to gabapentin seem to be rare. Only two cases of hypomanic episodes have been reported (Wong et al, 1997). Moreover, there is evidence that gabapentin has therapeutic effects in affective psychosis (Young et al, 1997). In the case reported here alternating or postictal hypomanic psychosis could be excluded. No other factors possibly contributing to interictal psychosis were detected. Thus, we feel that treatment with gabapentin is related to the patient's manic episode, even more so since she remained without any psychic abnormalities in a two-year follow-up. This may be in line with clinical observations on mood-elevating effects of gabapentin.

\section{Bech, R., Rafaelson, O. J., Kramp, P., et ol (1970) The mania rating scale: scale construction and inter- observer agreement. Neurophormocologx 17, 430-431. \\ Hauck, A. \& Bhaumilk, S. (1995) Hypomania induced by gabapentin. British Journal of Psychiatry, 167, 549.}

Short, C. A Cooke, L. (1995) Hypomania induced by gabapentin. British fournol of Psychiatry, 166, 679-680.

Wong, I. C. K., Tavernor, S. J. \& Tivernor, R. M. E. (1997) Psychiatric adverse effects of anticonvulsant drugs. Incidence and therapeutic implications. CNS Drugs, 8, 492-509.

Yound, L. T., Robb, J. C., Patcellaciots, L., of of (1997) Acute treatment of bipolar depression with gabapentin. Biological Psychiotry, 42, 851-853.

\section{F. M. Lewelce, J. Bawer, C. E. Elger}

Department of Epileptology, University of Bonn, Sigmund-Freud-Str. 25, D-53105 Bonn, Germany

\section{Polydactyly and functional psychosis}

Sir: I wish to comment on the report by Cardno et al (1998), which described five cases of co-occurrence of polydactyly and functional psychosis. I would like to report on two patients with polydactyly who also presented with mood disorders.

A 48-year-old married male had postaxial polydactyly on the right hand. There was no family history of polydactyly. His daughter had schizophrenia. At the age of 35 , he developed elated mood, grandiose delusions and other features suggesting a diagnosis of mania. The episode was successfully treated with neuroleptics for six months. He developed an episode of 